\title{
Doping profile measurement on textured silicon surface
}

\author{
Zahi Essa ${ }^{1}$, Nadjib Taleb ${ }^{1}$, Bernard Sermage ${ }^{1, *}$, Cédric Broussillou ${ }^{2}$, Barbara Bazer-Bachi ${ }^{2}$, and Maurice Quillec ${ }^{1}$ \\ 1 Probion Analysis, 37 rue de Fontenay, 92220 Bagneux, France \\ ${ }^{2}$ EDF ENR PWT, 33 Bd Saint Honoré, 38300 Bourgoin-Jallieu, France
}

Received: 28 July 2017 / Received in final form: 15 December 2017 / Accepted: 9 January 2018

\begin{abstract}
In crystalline silicon solar cells, the front surface is textured in order to lower the reflection of the incident light and increase the efficiency of the cell. This texturing whose dimensions are a few micrometers wide and high, often makes it difficult to determine the doping profile measurement. We have measured by secondary ion mass spectrometry (SIMS) and electrochemical capacitance voltage profiling the doping profile of implanted phosphorus in alkaline textured and in polished monocrystalline silicon wafers. The paper shows that SIMS gives accurate results provided the primary ion impact angle is small enough. Moreover, the comparison between these two techniques gives an estimation of the concentration of electrically inactive phosphorus atoms.
\end{abstract}

Keywords: textured surface silicon / ECV profiling / SIMS / PV emitter profiling

\section{Introduction}

Photovoltaic modules are becoming increasingly mainstream due to their fast decreasing price and the need for mankind to produce electrical energy without emitting large amounts of greenhouse gases in the atmosphere.

Silicon modules have been greatly improved in the last years so that the best efficiency is now above $24 \%$ while best cells have recently exceeded $26.6 \%$ for single junction $[1,2]$.

One of the very early optical improvement was to texture the surface of the silicon cells by creating random structures which reduce light reflection and increase photons penetration inside the silicon. For monocrystalline (100) wafers, the structures are pyramids formed by selective alkaline chemical etching leaving exposed the dense (111) atomic planes and thus forming square-based pyramids. The PN junction of most silicon photovoltaic cells is usually created by diffusion of an $n$-type dopant at high concentration (above $10^{20} \mathrm{~cm}^{-3}$ called the emitter) and a small depth $(<0.5 \mu \mathrm{m})$ in a lowly doped $\left(10^{14}-10^{16} \mathrm{~cm}^{-3}\right) p$-type substrate $[3,4]$. The space charge region (SCR) extends mainly into the $p$-type silicon due to the much higher carrier concentration in the emitter. The incoming photons are absorbed throughout the depth of the silicon wafer but the electron-hole pairs are mostly separated in the SCR. As recently reminded by several $\mathrm{PV}$ groups in a shared article (MIT, ISC, ISFH, et al., [5]); in order to reach state of the art $\mathrm{PV}$ efficiencies, it is necessary to optimize and thus to precisely characterize the $n$-type emitter doping profile of

\footnotetext{
* e-mail: bernard.sermage@probion.fr
}

the cells. The commonly used techniques for measuring doping profiles on flat wafers face some difficulties when it comes to analysing textured wafers. Those techniques include secondary ion mass spectrometry (SIMS), spreading resistance probe (SRP) and electrochemical capacitance voltage profiling (ECVP). Bevels cannot be made on textured surfaces and therefore SRP is to be excluded for textured wafers. SIMS can also be influenced by the textured surface geometry but our results show that major improvements can be made when the right abrasion angle is chosen. ECVP on the other hand is a well suited technique provided the right surface ratio is used [6,7]. In this paper, SIMS and ECVP techniques are compared in order to measure $n$-type profiles on several textured and polished wafers with phosphorus implanted and thermally activated emitters. SIMS measures all phosphorus atoms while ECVP measures electrically active donors which includes electrically active phosphorus atoms as well as some oxygen-related donors, the so-called thermal donors [8].

\section{Experimental}

\subsection{Samples preparation}

SIMS and ECVP measurements were carried out on four textured wafers and four polished wafers. The texture was obtained by etching the silicon substrate with an alkaline etching using among other chemicals a diluted $\mathrm{KOH}$ solution. For the (001) monocrystalline silicon wafer, this gives random pyramids i.e. with random sizes and random positions, but they all have the same orientation with (111) faces and square bases [9] as shown on Figure 1. 


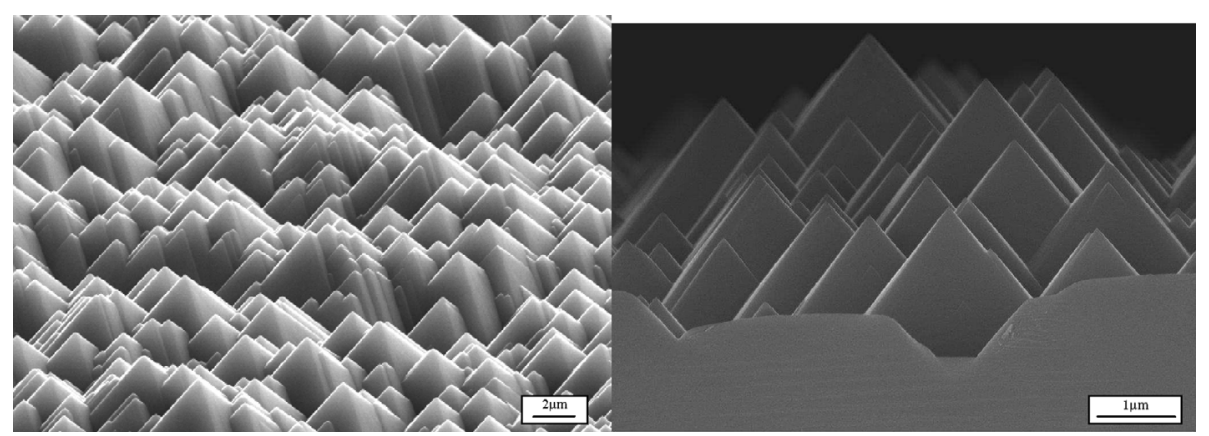

Fig. 1. Scanning electron microscopy images showing the pyramidal shape of the textured surface.

Table 1. Sample process conditions for polished (P1 to P4) and textured (T1 to T4) wafers using two phosphorus implantation doses (D1 and D2 $=1.25 \mathrm{D} 1)$ and two annealing durations at $850^{\circ} \mathrm{C}(\tau, 2 \tau)$.

\begin{tabular}{lll}
\hline \multicolumn{1}{c}{ Annealing conditions } & \multicolumn{2}{c}{ Implantation dose } \\
\cline { 2 - 3 } & \multicolumn{1}{c}{ D1 } & \multicolumn{1}{c}{$\mathrm{D} 2=1.25 \times \mathrm{D} 1$} \\
\hline None & P1 (Polished) T1 (Textured) & - \\
Standard time $\tau$ at $850^{\circ} \mathrm{C}$ & P2 (Polished) T2 (Textured) & - \\
Double time $2 \tau$ at $850^{\circ} \mathrm{C}$ & P3 (Polished) T3 (Textured) & P4 (Polished) T4 (Textured) \\
\hline
\end{tabular}

The same set of implantation and annealing conditions was applied for both textured and polished wafers. Two implantation doses were used: D1 and D2 with D2 $=1.25 \mathrm{D} 1$. Two annealing conditions were used with the high temperature step at $850^{\circ} \mathrm{C}$ for a standard time $\tau$ and double the time $2 \tau$. A non-annealed sample was also characterized by SIMS to know as-implanted profile. Process conditions are summarised in Table 1 . Note that samples Pi and Ti received the same process (called i). The only difference is the polished or textured surface.

\subsection{SIMS measurements}

The SIMS profiles were carried out on a CAMECA 4F equipment, using $\mathrm{O}_{2}{ }^{+}$primary ions and positive secondary ions detection, in high resolution mode in order to avoid mass interference between ${ }^{31} \mathrm{P}$ and ${ }^{30} \mathrm{Si}^{1} \mathrm{H}$. Oxygen flooding was also used in the experiments for a higher phosphorus ionisation rate and a lower surface roughening during the analysis. Pressure was $5.10^{-5}$ Torr and the primary ions current was $1 \mu \mathrm{A}$. SIMS equipment with a magnetic sector architecture is limited by the primary off normal impact angle $\alpha$ versus the [001] direction normal to the wafer which leads to different etching rates of the faces of the pyramids depending on their orientations relative to the ion beam direction. The phosphorus concentration is obtained by the ratio between phosphorus and silicon signals. A comparison with standard gives the relative sensitivity factor. The incident angle $\alpha$ of the primary ion beam can be varied by modifying the SIMS primary and secondary acceleration voltages. Three incident angles were used: $36^{\circ}, 42^{\circ}$ and $64^{\circ}$ with the energy $12.5 \mathrm{keV}, 5.5 \mathrm{keV}$ and $2 \mathrm{keV}$.

\subsection{ECVP measurement details}

ECVP profiles give the concentration of electrically active dopants, mainly phosphorus substitutional atoms and the difference between SIMS and ECVP profiles gives the concentration of inactive phosphorus. These inactive phosphorus atoms can be interstitial $\mathrm{P}$ or clusters of $\mathrm{P}$. They induce deep levels in the bandgap which increase Shockley, Read, Hall (SRH) recombination and lower the efficiency of photovoltaic cells $[5,10]$.

ECVP measurements were performed on the 6 annealed samples. ECVP [6] is an isotropic measurement and should work as well on textured and polished surfaces. The BioRad PN4300PC equipment was used for these measurements. The sample surface is in contact with an electrolyte containing half $0.1 \mathrm{M} / \mathrm{l} \mathrm{NH}_{4} \mathrm{FHF}$ and half $0.5 \mathrm{M} / 1 \mathrm{HCl}$. The electrolyte-semiconductor interface acts as a Schottky junction. The equipment uses a double modulation to measure the capacitance $C$ of the Schottky junction and its derivative $d \mathrm{C} / d \mathrm{~V}$. The doping concentration $N$ at the edge of the SCR is given by $[7,11,12]$ :

$$
N=\frac{C^{3}}{\varepsilon_{r} \varepsilon_{0} q A^{2} \frac{\partial C}{\partial V}}
$$

where $\varepsilon_{r}$ is the permittivity relative to vacuum, $\varepsilon_{0}$ is the vacuum permittivity, $q$ is the electron charge and $A$ is the silicon area in contact with the electrolyte.

When silicon is positively polarised, the anions of the electrolyte $\left(\mathrm{Cl}^{-}, \mathrm{F}^{-}\right)$move toward the silicon surface and etch a certain amount of material proportional to the current integral. The depth $d$ where the doping level is 

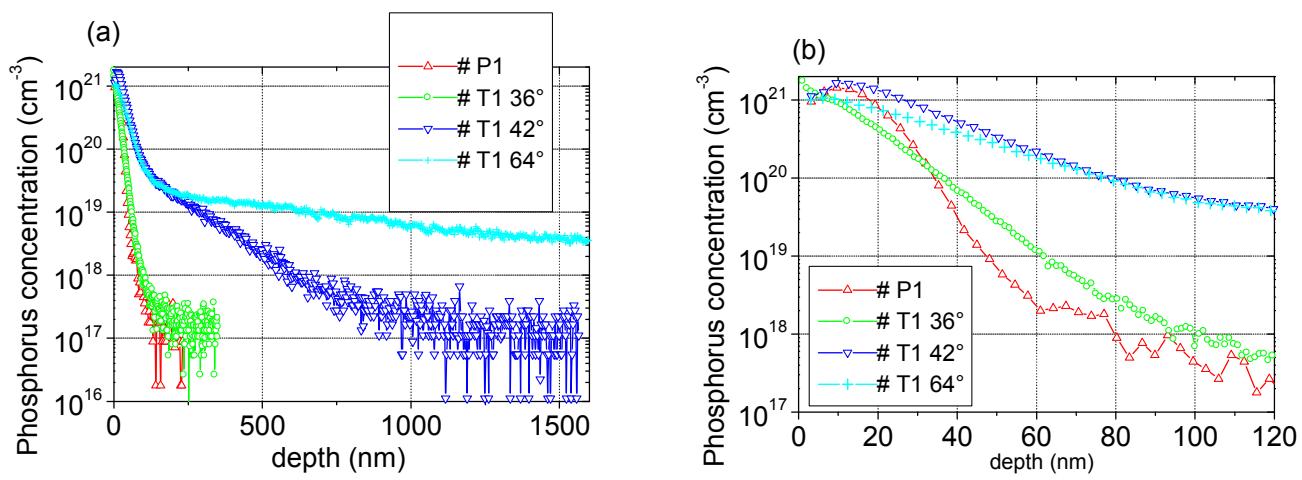

Fig. 2. Comparison of SIMS profiles in sample 1 with a polished and textured surface. In the case of the textured wafer, the results are given for 3 different incident angles of the primary ions: $36^{\circ}, 42^{\circ}, 64^{\circ}$. Figure $2 \mathrm{~b}$ is an enlargement of the first nanometres.

measured, is given by [11]:

$$
d=d_{\mathrm{etch}}+d_{\mathrm{dep}}=\frac{M}{z F \rho A} \int I d t+\frac{\varepsilon_{r} \varepsilon_{0} A}{C},
$$

where $d_{\text {etch }}$ is the etched depth, $d_{\text {dep }}$ is the depletion layer depth, $M$ is the molecular mass, $z$ is the effective silicon valence which is taken to be 3.7 [11], $F$ is Faraday's constant and $\rho$ is silicon density.

This allows to make successive etchings on the sample followed by capacitance measurements in order to obtain the doping profile in the semiconductor.

As can be seen from equations (1) and (2), the doping level and the etched depth depend on the area in contact with the electrolyte. As said previously, due to the pyramidal textured surface, the theoretical ratio between the surface in contact with the electrolyte and the macroscopic sample surface $f$ is $\sqrt{ } 3=1.73$. This value was experimentally confirmed in ECVP measurements by Bock et al. [6]. Hinken et al. [13] have shown that the $f$ value should be 1.73 when the depleted thickness is small compared to the texture height and decreases when the depleted thickness increases. In our case, the depleted thickness is small compared to the texture height (a few microns) so we use $f=1.73$. After the ECVP measurements, the depth and the width of the craters were obtained with a mechanical profilometer for polished wafers. The depth and doping values of the profile are then corrected using equations (1) and (2). In the case of the textured wafers, the mechanical profilometer could not be used due to the high surface roughness. We have thus assumed that the microscopic etching rate (for the same microscopic current density) and the width of the crater of the textured samples were the same as the ones for the polished wafers.

\section{Results and discussion}

\subsection{SIMS results}

The phosphorus profiles measured by SIMS on sample P1 and on sample T1 with the three incident angles $\left(36^{\circ}, 42^{\circ}\right.$ and $64^{\circ}$ ) are given on Figure 2.
Figure 2a shows that for a $36^{\circ}$ incident angle of the primary ion beam, the profile on the textured sample is very similar to that on the polished sample. It is not the case for the larger incident angles $\left(42^{\circ}\right.$ or $\left.64^{\circ}\right)$ where the profiles show a long tail which we attribute to the "shadow effect" which means that, due to the incident angle of the ion beam, the opposite face of the pyramids is not etched by the primary ion beam. Thus, there will remain on the sample some areas with high doping which are still slowly etched at the end of the profile and will artificially increase the measured doping level in this part of the profile. This is particularly important in the case of wafers with high doping at the surface followed by much lower doping deeper in the material (i.e. high doping gradient) which is the case of sample $\mathrm{T} 1$ where the doping is $10^{21} \mathrm{~cm}^{-3}$ near the surface and decreases by 4 orders of magnitude at $150 \mathrm{~nm}$.

Let us note $\theta_{i}$ the angle between the incident beam and the normal to the $i$ th face as shown on Figures $3 \mathrm{a}$ and $3 \mathrm{~b}$. The shadow effect has two origins: on the one hand, the face opposite to the incoming ion beam will not be etched if $\theta$ is larger than $90^{\circ}$. This is known as the "form shadow" which is the shadow that is found on the object itself and that depends on the form of the object (here a pyramid) and its orientation relative to the incoming beam. On the other hand, part of the faces of the other surrounding pyramids may be partly hidden due to a cast shadow from the previous pyramid.

The azimuthal direction of the incident beam relative to the pyramid faces during the SIMS measurement is not known with precision, thus we consider that the incident beam plane makes an angle $\phi$ with the (010) plane as shown on Figure $3 \mathrm{~b}$.

Using the scalar product between the unit vector of the SIMS incident beam directed from the sample toward the ion source: $(\sin \alpha \cos \phi ; \sin \alpha \sin \phi ; \cos \alpha)$ and the normal to the four faces, the $\theta_{i}$ angles are:

$$
\begin{aligned}
& -\cos \theta_{1}=(\sin \alpha(\cos \phi+\sin \phi)+\cos \alpha) / \sqrt{ } 3 ; \\
& -\cos \theta_{2}=(\sin \alpha(\sin \phi-\cos \phi)+\cos \alpha) / \sqrt{ } 3 ; \\
& -\cos \theta_{3}=(-\sin \alpha(\cos \phi+\sin \phi)+\cos \alpha) / \sqrt{ } 3 ; \\
& -\cos \theta_{4}=(\sin \alpha(\cos \phi-\sin \phi)+\cos \alpha) / \sqrt{ } 3 .
\end{aligned}
$$

For example, in the case of $\phi=45^{\circ}$, i.e. the incident plane contains the normal to the (111) plane, the angles between the incident beam and the normal to each of the 

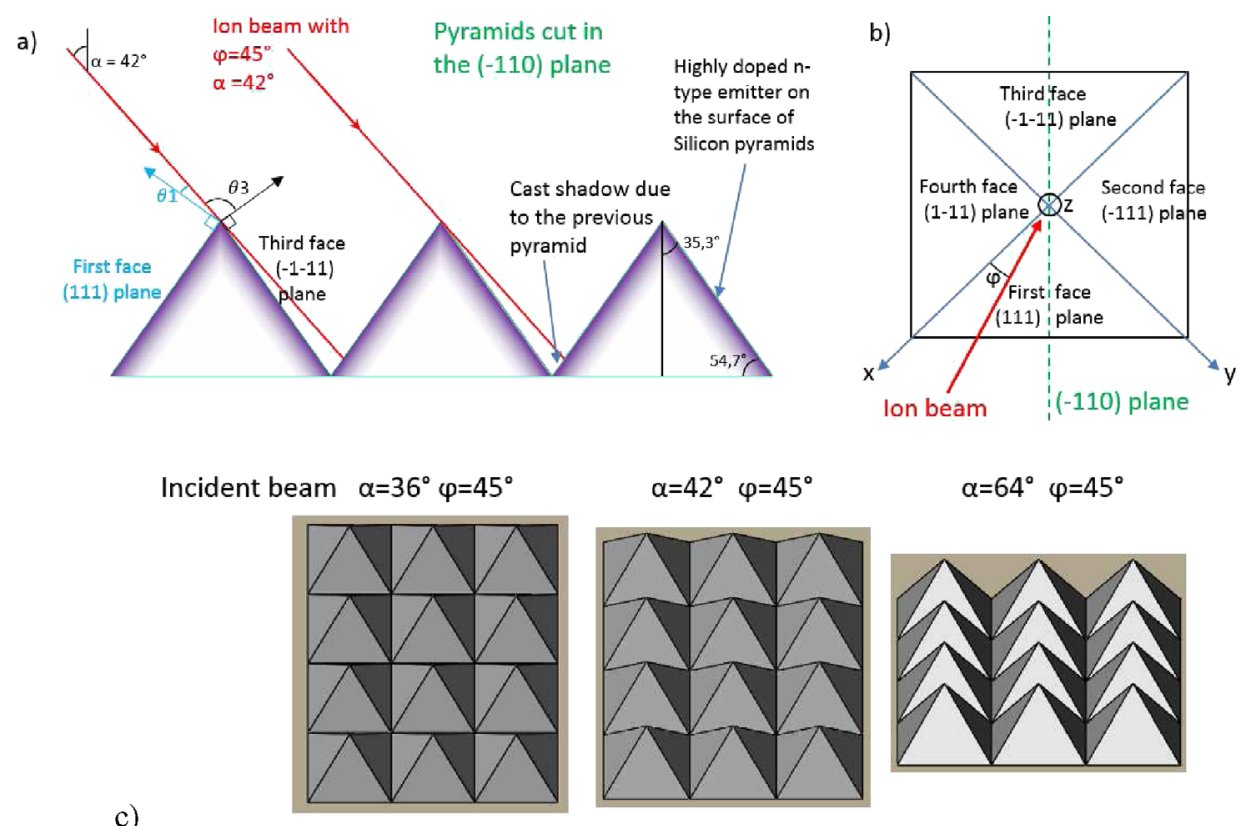

Fig. 3. (a) Diagram in the $(-110)$ incident plane showing pyramids of the textured surface and the incident primary ion beam, in the case where the incident plane contains the [111] direction. (b) Projection in the sample plane of the pyramid and of the incident ion beam. (c) 3D views of the pyramids as a function of the ion beam incident angle.

Table 2. Angles between the incident ion beam and the normal to the four pyramids' planes for $\phi=45^{\circ}$.

\begin{tabular}{llll}
\hline \multirow{2}{*}{$\begin{array}{c}\text { Ion beam } \\
\text { incidence angle } \alpha\end{array}$} & \multicolumn{3}{c}{$\begin{array}{c}\text { Angle between incident ion beam } \\
\text { and normal to the pyramid planes }\end{array}$} \\
\cline { 2 - 4 } $36^{\circ}$ & $\theta_{1}$ & $\theta_{2}=\theta_{4}$ & $\theta_{3}$ \\
\cline { 2 - 4 } $42^{\circ}$ & 18.7 & 62.2 & 90.7 \\
$64^{\circ}$ & 12.7 & 64.6 & 96.7 \\
\hline
\end{tabular}

four faces of the pyramids are given in Table 2 for the three experimental $\alpha$ angles $\left(36^{\circ}, 42^{\circ}, 64^{\circ}\right)$. The case for $\phi=45^{\circ}$ is interesting as it is the condition for which the "form shadows" as well as the "cast shadows" are the largest and thus constitute a worst-case scenario for the shadowing.

For this azimuthal angle $\phi=45^{\circ}$, the face opposite to the incident beam (third face in Fig. 3b)) is not etched because the incident beam makes an angle larger than $90^{\circ}$ with the normal to this $(-1-11)$ face. One can show that, for $\alpha=36^{\circ}$, angle $\theta_{3}$ is maximum at $90.7^{\circ}$ for $\phi=45^{\circ}$ and stays larger than $90^{\circ}$ for $\phi$ between $31.7^{\circ}$ and $58.3^{\circ}$. In the case of $\alpha=42^{\circ}, \theta_{3}$ is larger than $90^{\circ}$ for $\phi$ between $6.7^{\circ}$ and $83.2^{\circ}$. Since we observe a tail on the profile for $\alpha=42^{\circ}$ and not for $36^{\circ}$, the azimuthal angle is between $6.7^{\circ}$ and $31.7^{\circ}$. Let us note that the most favourable case is for $\phi=0$, when the ion beam is in the same vertical plane as the edges of the pyramids. In this case, $\theta_{3}$ is smaller than $90^{\circ}$ for $\alpha<45^{\circ}$ which can be easily obtained with the SIMS. A better ion milling homogeneity on all four faces could also be reached if the sample were to be rotated around the normal to its plane during the measurement ${ }^{1}$.
The second shadow effect (cast shadow) is in fact the same as the form shadow since if the ion beam can etch the four faces of a pyramid, no shadow will be cast on the surrounding pyramids.

The profile on Figure 2a for a textured wafer measured with an incident angle of $36^{\circ}$, is similar to that of the polished one but does not perfectly reproduce it (Fig. 2b). The phosphorus concentration on the near surface region (between 5 and $30 \mathrm{~nm}$ ) is higher for the polished wafer than for the textured one. After the first $30 \mathrm{~nm}$, the concentration is larger in the textured one (Fig. 2b). This is the combination of two effects related to the implantation and the SIMS technique itself: 1) The implantation direction is normal to the surface and the same dose per unit area of the sample is applied for the textured and the polished sample. Due to the pyramids, the developed surface area in the textured sample is increased by a factor $f=\sqrt{ } 3=1.73$ compared to the polished wafer thus the doping concentration per unit developed area should be decreased in the textured sample by the same factor. 2) Shadowing effect in SIMS as seen previously. Due to the first effect, the profile on the textured sample should be lower by the factor 1.73 in comparison to the polished one. On Figure 2b, a lower concentration on the textured is only observed for depths between 5 and $30 \mathrm{~nm}$ but the ratio is smaller than 1.73 . Beyond $30 \mathrm{~nm}$, the profile on the textured sample is higher. These two points are due to the steep profile and to the fact that the ion beam makes a large angle (between $83^{\circ}$ and $90^{\circ}$ )

\footnotetext{
$\overline{1}$ The solution to rotate the sample around an axis normal to the sample so that the four faces of the pyramids receive the same duration of incident ion beam is proposed by Cameca but has not been tested and would not suppress all shadowing in particular for large incident angles such as $64^{\circ}$.
} 


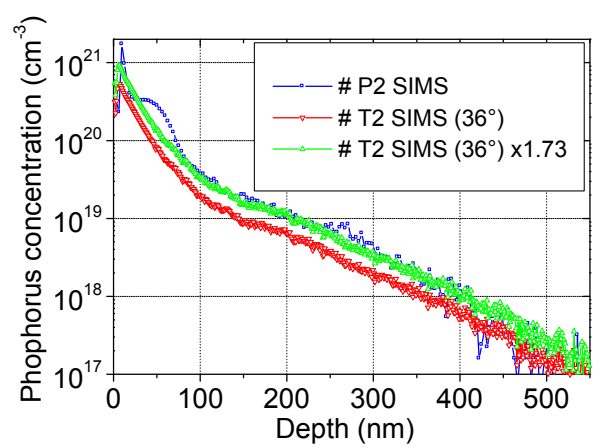

Fig. 4. Phosphorus profile measured by SIMS on sample P2 and T2 (standard annealing) with a polished and textured surface. For sample T2, the incident ion beam makes an angle of $36^{\circ}$ with the normal to the wafer. The green curve shows the profile on the textured sample multiplied by 1.73 .

with the third pyramid face so that this face is not etched with the same speed as the other ones. Finally, SIMS profile measurement with an incident primary beam angle of $36^{\circ}$ gives an accurate but yet not perfect approximation of the emitter profile in implanted not annealed textured wafers.

In the case of the annealed samples, the phosphorus concentration gradient is less steep due to the diffusion during the annealing time at high temperature. Thus the effect of the different etching rates on the profile is smaller and the phosphorus profile on the textured sample follows that of the polished sample but lowered by the surface ratio $f$. This is what we observe on Figure 4 where the phosphorus profile on the textured sample multiplied by 1.73 is identical to that of the polished sample except on the 70 first nanometers where the polished profile is higher which we do not explain. This also confirms that the area ratio is equal to the theoretical value: $f=1.73$.

\subsection{Comparison of ECVP and SIMS on polished and textured wafers}

Figure 5 shows the comparison of SIMS and ECVP profiles in sample T2 (textured, standard implantation dose and annealing time). Profiles are equal between 40 and $300 \mathrm{~nm}$ which shows that all phosphorus atoms are electrically active. In the ECVP profile, we use for the factor $f$, the value 1.73. A smaller $f$ value would have put the ECVP concentration above the phosphorus concentration measured by SIMS which is not possible so we confirm the value $f=1.73$ in ECVP measurements.

Below $40 \mathrm{~nm}$, the SIMS concentration is larger than the ECVP one. This shows that there are inactive phosphorus atoms and thus probably recombination centres near the surface but this is not limiting since the hole concentration is reduced at the surface and the carrier recombination will stay low. Conversely, the high phosphorus concentration at the surface is needed to obtain a good electric contact with the screen-printed metallic fingers. Beyond $350 \mathrm{~nm}$, the SIMS concentration is again larger than ECVP. This is partly due to the "remaining shadowing effect" since the large incident angle on the third face lower the etching rate.

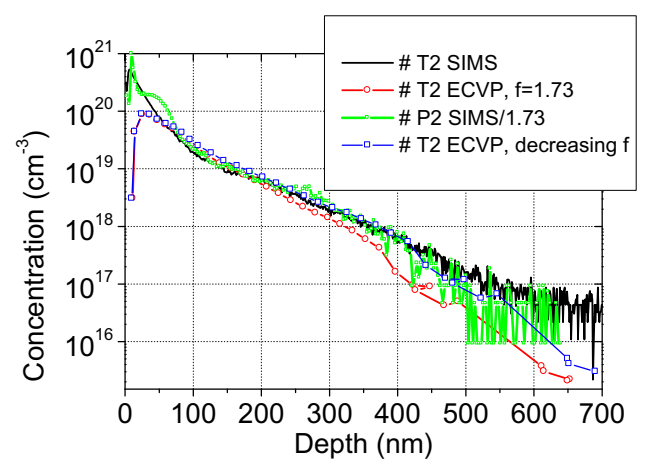

Fig. 5. Comparison between ECVP and SIMS profiles in textured sample T2. SIMS measurement on the textured sample was done with an incident angle of $36^{\circ}$ for the primary ions. Also shown the SIMS profile on the polished sample P2 divided by 1.73 and the ECVP profile with an $f$ factor decreasing along the profile.

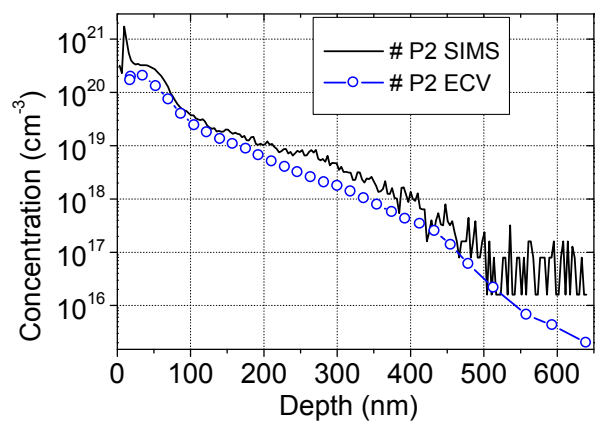

Fig. 6. Comparison of ECVP and SIMS profiles in the polished (P2) wafer.

On Figure 5 is also shown the SIMS profile on the polished sample divided by 1.73 which we think is the true phosphorus profile in the textured sample. There is a remaining difference between SIMS and ECVP profiles after $300 \mathrm{~nm}$. We think that it is due to a smoothing of the pyramidal structures during the electrolytic etching. When we look to the sample after the ECVP, we observe that the bottom of the crater is brighter than the unetched part which means that the albedo is larger and that the pyramids have partly disappeared. We show also on Figure 5 an ECVP profile with a decreasing $f$ value:

$$
f=1+0.73 \times \exp \left(-d / d_{0}\right),
$$

where $d$ is the depth and $d_{0}$ is a characteristic depth which is $1100 \mathrm{~nm}$ for sample T2. This ECVP profile is in good agreement with the SIMS profile on the polished wafer divided by 1.73. So we don't think that there is a difference between the concentration of phosphorus atoms and the $n$ doping and that there are electrically inactive phosphorus atoms in this part of the profile.

Figure 6 shows that for the polished sample P2, the ECVP concentration curve lies slightly below the SIMS profile. A similar behaviour was observed by Steyer et al. [10] and Wagner et al. [5] and is attributed to phosphorus atoms which are present in the silicon but are not electrically active. These inactive phosphorus are typically 


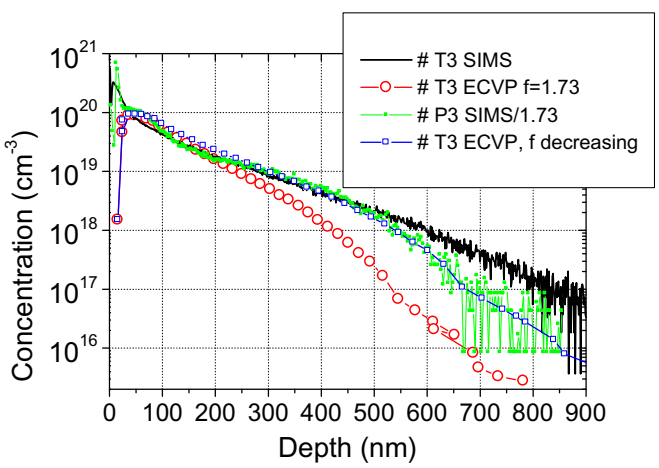

Fig. 7. Comparison of SIMS and ECVP profiles in the textured wafer T3. SIMS measurement was performed with an incident angle of $36^{\circ}$ for the primary ions. Also shown, the SIMS profile on the polished wafer P3 divided by 1.73 and an ECVP curve with a $f$ coefficient decreasing along the profile.

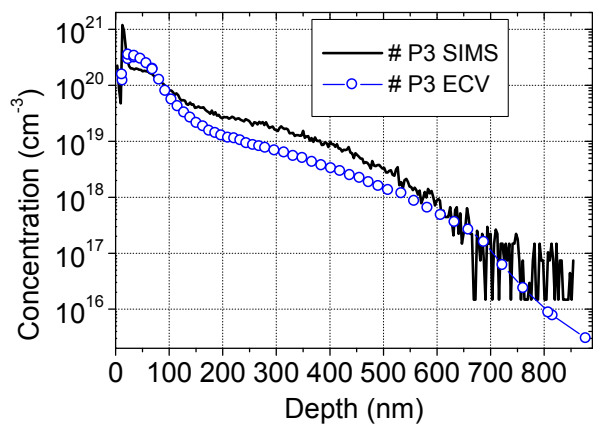

Fig. 8. Comparison of SIMS and ECVP profiles in wafer P3 with a polished surface and a longer annealing time.

interstitial phosphorus or clusters and are recombination centres as shown on their influence on saturation current $J_{0}$ in photovoltaic devices [5]. As shown by these authors, this occurs all the more when phosphorus concentration at the surface is high $\left(\geq 10^{21} \mathrm{~cm}^{-3}\right)$. This is not observed for the textured sample because the microscopic surface concentration is only $7.10^{20} \mathrm{~cm}^{-3}$ due to the larger specific area while it is $1.7 \times 10^{21} \mathrm{~cm}^{-3}$ in the polished one. Thus, the same macroscopic implanted dose does not yield the same electrically active dopant profile whether it is deposited on a polished or textured wafer. The "standard" dose D1 used for this experiment is already too high for polished wafers with a standard annealing and induces inactive phosphorus deep in the silicon.

Figures 7 and 8 show the profiles corresponding to textured and polished wafers for process conditions 3 (T3 and P3). These samples were implanted with a standard dose but have been annealed for a longer time compared to condition 2 (Tab. 1), so that phosphorus atoms have diffused deeper inside the silicon. As in sample polished P2, there are inactive phosphorus atoms between 150 and $550 \mathrm{~nm}$ in the polished sample due to the too high implanted dose (Fig. 8). In the textured one, ECVP and SIMS concentrations are equal between 50 and $250 \mathrm{~nm}$. After $250 \mathrm{~nm}$, ECVP concentration decreases much more

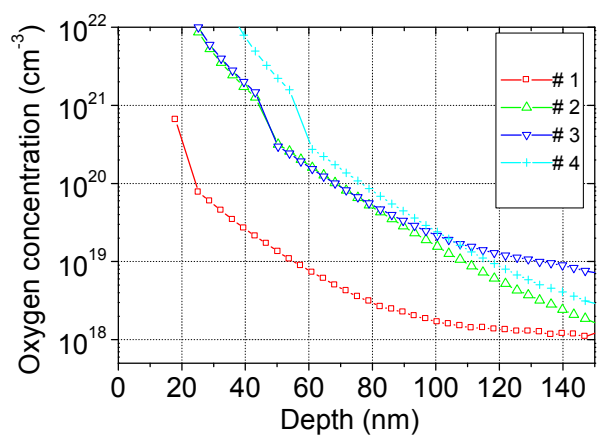

Fig. 9. Oxygen SIMS profile on wafers 1-4 showing diffusion of oxygen inside the silicon. Implantation dose and annealing conditions as described in Table 1 .

than the SIMS one. As in sample T2, the difference between SIMS and ECVP is partly explained by a remaining shadowing effect as can be seen with the polished wafer (P3) SIMS profile divided by 1.73. Another part is due to a smoothening of the pyramidal texture as shown by the blue curve with a $f$ factor decreasing along the profile as in equation (3). The characteristic depth is here $d_{0}=750 \mathrm{~nm}$. As in sample 2, we think there are no electrically inactive phosphorus atoms in this part of the profile at a depth $>250 \mathrm{~nm}$ in the silicon.

Let us note that near the surface between 20 and $70 \mathrm{~nm}$, ECVP measured doping is larger than phosphorus concentration in the polished wafer (Fig. 8). This is unexpected and could be due to the presence of oxygen that has diffused from the thermal oxide inside the silicon during annealing. Oxygen is a "thermal donor" in silicon $[8,14,15]$ which can influence the value of the measured electrical activity in ECVP and leads to an overestimation of the $n$-type dopant profile in the zone where oxygen is in high concentration.

The presence of oxygen in silicon is confirmed by the oxygen SIMS profiles using Cs primary ions on samples 1-4 on Figure 9 (the profiles are similar on textured and polished wafers). The figure shows that the amount of oxygen is much higher after thermal annealing than before (P1 before annealing vs P2-P4 after annealing). Oxygen diffuses inside the wafer during the high temperature step and oxygen concentration is higher than $10^{20} \mathrm{~cm}^{-3}$ in the first $70 \mathrm{~nm}$ close to the surface. This high oxygen concentration in the first tens of nanometres measured by SIMS can explain the ECVP curve being above the SIMS phosphorus concentration in this region (depth $<70 \mathrm{~nm}$ ).

Figure 10 shows the profiles corresponding to samples $\mathrm{T} 4$ and $\mathrm{P} 4$ with an implantation dose increased by $25 \%$ and same annealing as T3 and P3. Although the implanted dose is larger, the total phosphorus concentration in the polished wafer after annealing measured by integrating the SIMS profile is similar to that of wafers P2 and P3. It is likely that the extra phosphorus over the solubility limit implanted in P4 compared to P3 outgassed during the first minutes of the annealing while the thermal oxide had not been formed yet to retain this phosphorus source. On the polished sample $\mathrm{P} 4$, we observe inactive phosphorus atoms between 200 and $700 \mathrm{~nm}$ as for previous P2 and P3 samples and a 

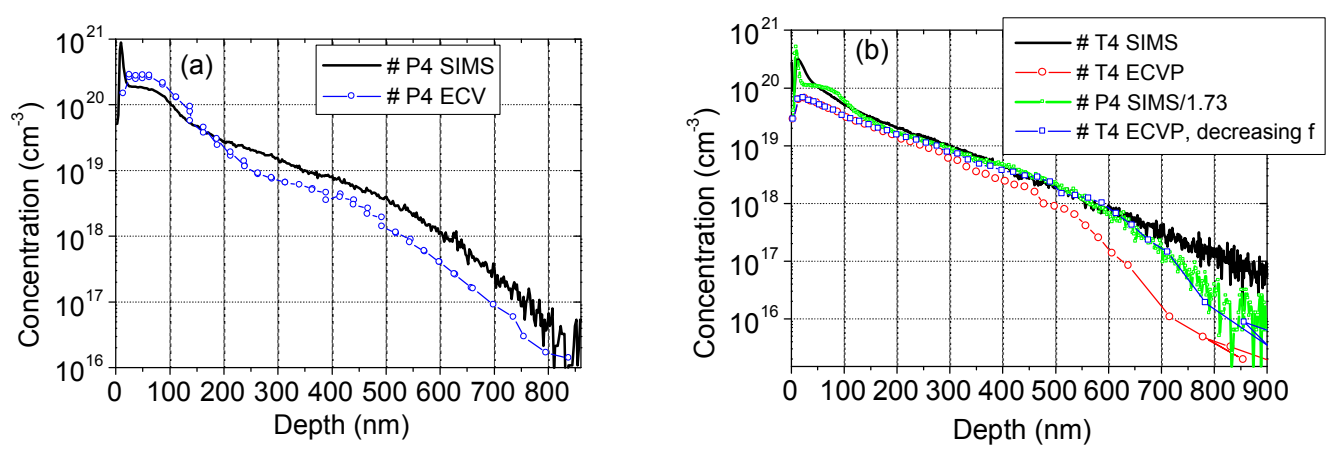

Fig. 10. Comparison of SIMS and ECVP profiles in flat and textured wafer 4. SIMS measurement on the textured sample was done with an incident angle of $36^{\circ}$ for the primary ion beam. Also shown on Figure $8 \mathrm{~b}$ the SIMS profile on the polished sample divided by 1.73 and the ECVP profile with a $f$ factor decreasing along the profile.

Table 3. Sheet resistance: measured value and value calculated from ECVP profiles.

\begin{tabular}{lll}
\hline Wafer & Measured $R \square(\Omega)$ & $\begin{array}{c}R \square(\Omega) \text { calculated } \\
\text { from ECVP profile }\end{array}$ \\
\hline P2 & 58 & 56 \\
T2 & 69 & 95 \\
P3 & 39 & 37 \\
T3 & 52 & 58 \\
P4 & 33 & 29 \\
T4 & 43 & 64 \\
\hline
\end{tabular}

larger ECVP concentration near the surface which we attribute to oxygen. On the textured sample, SIMS and ECVP concentrations are similar between 100 and $450 \mathrm{~nm}$. For depth larger than $450 \mathrm{~nm}$, the difference between SIMS and ECVP can be explained by a mild remaining shadowing effect in SIMS and by the decrease of $f$ factor using equation (3) and $d_{0}=750 \mathrm{~nm}$ for ECVP.

We have measured the sheet resistance $R \square$ on the polished and textured samples. For polished wafers, the results (Tab. 3) are in good agreement with those calculated from ECVP profiles using Masetti's mobility [16]. In the case of the textured wafers, $R \square$ calculated from the ECVP profile is larger than the 4 points measured value. We think that the relationship between the dopant profile and the 4 points measured $R \square$ is not straightforward in textured wafers as it is in polished wafers due to effects linked with the surface geometry which are not taken into account in this electrical model.

\section{Conclusion}

We have shown that SIMS with an incident angle of $36^{\circ}$ for the primary ion beam is a good technique for measuring emitter dopant profiles in textured silicon wafers. It is favourable that the incident ion beam is in the same plane as the ridge of the pyramids (010). For this incident primary ion beam orientation, the angle of the beam versus the normal to the sample must be smaller than $45^{\circ}$ and preferably close to $36^{\circ}$ to suppress the tail. In the case of annealed implanted silicon wafers, the phosphorus profile on the textured wafers is the same as that of polished wafer divided by the geometrical factor $f$ due to the fact that the textured wafer has a larger specific area. The measured $f$ value is equal to the theoretical value obtained for pyramids with a square base and (111) faces: $f=\sqrt{ } 3=1.73$.

The comparison with ECVP enable to estimate the presence of electrically inactive phosphorus atoms.

For the polished wafers, for the three process conditions, there are inactive phosphorus atoms probably present due to a too high implantation dose for flat surfaces.

For the textured wafers, ECVP provides sensible doping profiles compared to SIMS provided that we take into account that the area in contact with the electrolyte is the one delimited by the $\mathrm{O}$ ring multiplied by the area factor $f$. At the beginning of the profile, $f$ is equal to 1.73 (due to the pyramidal texture) and it decreases along the profile because the chemical etching during ECVP smoothens the pyramids.

The authors thank Pierre-Philippe Grand and Jessica Legendre from IPVF (Ile-de-France Photovoltaic Institute) for SEM images and Nicolas Jarru at Photowatt for help in the 3D drawings of the pyramids. Z. Essa, N. Taleb, B. Sermage and M. Quillec gratefully acknowledge French Research Minister for the support with CIR contract.

\section{References}

1. M.A. Green et al., Solar cell efficiency tables (version 50), Prog. Photovolt. Res. Appl. 25, 668 (2017)

2. New World Record Established for Conversion Efficiency in a Crystalline Silicon Solar Cell - Conversion Efficiency of 26.63\% Achieved in a Practical Cell Size, Press release, 2017, http://www.kaneka.co.jp/en/service/news/nr201708252

3. V.V. Iyengar, B.K. Nayak, M.C. Gupta, Silicon PV devices based on a single step for doping, anti-reflection and surface passivation, Sol. Energy Mater. Sol. Cells 94, 2205 (2010)

4. M.A. Green, The path to $25 \%$ silicon solar cell efficiency: history of silicon cell evolution, Prog. Photovolt. Res. Appl. 17, 183 (2009)

5. H. Wagner et al., Optimizing phosphorus diffusion for photovoltaic applications: Peak doping, inactive phosphorus, gettering, and contact formation, J. Appl. Phys. 119, 185704 (2016) 
6. R. Bock, P.P. Altermatt, J. Schmidt, Accurate extraction of doping profiles from electrochemical capacitance voltage measurements, in Proc. 23rd Eur. Photovolt. Sol. Energy Conf. Exhib., Valencia, Spain, 2008, pp. 1510-1513.

7. Y. Komatsu, et al., Calibration of electrochemical capacitance-voltage method on pyramid texture surface using scanning electron microscopy, Energy Procedia 38, 94 (2013)

8. F. Jay, J. Veirman, M. Tomassini, $10 \mathrm{~ms}$ carrier lifetimes on thermal donor-doped Cz wafers: application to heterojunction solar cells, in the 29th European PV Solar Energy Conference and exhibition proceedings of the international conference held in Amsterdam, The Netherlands, 2014, pp. 798-803

9. X. Wang, M.A. Alam, Estimating pyramid density of a random-textured surface by capacitance-voltage measurement of c-Si solar cells, in Proc. 39th Photovoltaic Specialists Conference, Tampa Florida USA, 2013, pp. 1-5.

10. M. Steyer et al., New method for determination of electrically inactive phosphorus in $n$-type emitters, Energy Procedia 77, $316(2015)$
11. E. Peiner, A. Schlachetzki, D. Kruger, Doping profile analysis in si by electrochemical capacitance-voltage measurements, J. Electrochem. Soc. 142, 576 (1995)

12. B. Sermage et al., Electrochemical capacitance voltage measurements in highly doped silicon and silicon-germanium alloys, J. Appl. Phys. 119, 155703 (2016)

13. D. Hinken et al., Determination of the base-dopant concentration of large-area crystalline silicon solar cells, IEEE Trans. Electron Devices 57, 2831 (2010)

14. G. Pensl Proc, in 5th Int. School ISPPME (1987)

15. Data in science and technology, Semiconductors, Group IV and III-V, Madelung, (Springer, Berlin, 1991), p. 25

16. G. Masetti, M. Severi, S. Solmi, Modeling of carrier mobility against carrier concentration in arsenic-, phosphorus-, and boron-doped silicon, IEEE Trans. Electron Devices 30, 764 (1983)

Cite this article as: Zahi Essa, Nadjib Taleb, Bernard Sermage, Cédric Broussillou, Barbara Bazer-Bachi, Maurice Quillec, Doping profile measurement on textured silicon surface, EPJ Photovoltaics 9, 5 (2018) 